This is a provisional PDF only. Copyedited and fully formatted version will be made available soon.

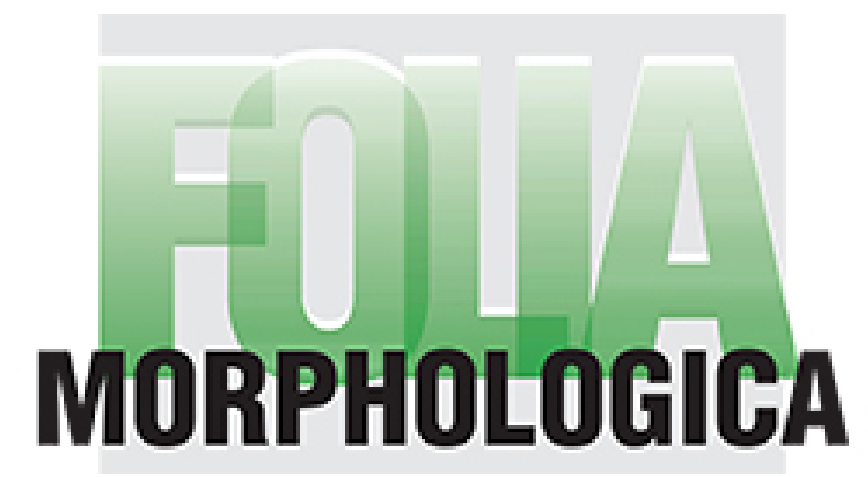

ISSN: 0015-5659

\title{
Morphological classification and measurement of the glenoid cavity using three-dimensional reconstruction in a Chinese population
}

Authors: Y. Chen, J. Xiong, W. Chen, D. Xie, Y. Zhang, Y. Mo, X. Gu, L. Zhang

DOI: $10.5603 / F M . a 2022.0017$

Article type: Original article

Submitted: $2021-11-30$

Accepted: $2022-02-03$

Published online: 2022-02-17

This article has been peer reviewed and published immediately upon acceptance.

It is an open access article, which means that it can be downloaded, printed, and distributed freely, provided the work is properly cited.

Articles in "Folia Morphologica" are listed in PubMed. 
Morphological classification and measurement of the glenoid cavity using threedimensional reconstruction in a Chinese population

Y. Chen et al., Morphological classification and measurement of the glenoid cavity using 3D reconstruction in a Chinese population

Y. Chen ${ }^{1}$, J. Xiong ${ }^{2}$, W. Chen ${ }^{1}$, D. Xie ${ }^{1 *}$, Y. Zhang ${ }^{1}$, Y. Mo ${ }^{1}$, X. Gu ${ }^{1}$, L. Zhang ${ }^{3,4,5,6}$

${ }^{1}$ Department of Orthopedics, Chinese and Western Medicine Hospital of Yibing, Yibing, China

${ }^{2}$ School of Clinical Medicine, Southwest Medical University, Luzhou, China

${ }^{3}$ Department of Orthopedics, The Affiliated Traditional Chinese Medicine Hospital of Southwest Medical University, Luzhou, China

${ }^{4}$ Center for Orthopedic Diseases Research, Affiliated Traditional Chinese Medicine Hospital of Southwest Medical University, Luzhou, China

${ }^{5}$ Expert Workstation in Luzhou, Luzhou, China

${ }^{6}$ Clinical Base of Affiliated Traditional Chinese Medicine Hospital of Southwest Medical University, Guangdong Province Medical 3D Printing Application

Transformation Engineering Technology Research Center, Luzhou, China

Address for correspondence: Dr. Xin-ke Gu, Department of Orthopedics, Chinese and Western Medicine Hospital of Yibing, e-mail: nxgxk123@163.com; Dr. Lei Zhang, Affiliated Traditional Chinese Medicine Hospital of Southwest Medical University, Luzhou, China, 646000., e-mail: zhanglei870722@126.com

*These authors contributed equally to this work. 
Yong-hong Chen, Ji-xiang Xiong, Wen Chen, Dong-bo Xie are the co-first authors.

\section{ABSTRACT}

Background: The purpose of this study was to examine the various shapes and record the morphometric data of the glenoid cavity in a Chinese population.

Materials and methods: A total of 501 scapulae, 247 left and 254 right, were analyzed. We classified the shape of the glenoid cavity as type I (pear-shaped), type II (oval-shaped), type III (teardrop-shaped), type IV (calabash-shaped) or type V (inverted comma-shaped). Four defined parameters, the superior-inferior glenoid diameter (AB), upper anterior-posterior glenoid diameter (CD), lower anteriorposterior glenoid diameter (EF) and glenoid index (GI), were measured, and five shapes were classified via three-dimensional reconstruction.

Results: The mean AB, CD, EF and GI values of the glenoid were $3.51 \pm 0.41 \mathrm{~cm}$, $1.95 \pm 0.28 \mathrm{~cm}, 2.60 \pm 0.34 \mathrm{~cm}$, and $1.35 \pm 0.12 \mathrm{~cm}$, respectively. The AB value of type II glenoid cavities was significantly smaller than that of type I and III glenoid cavities ( $\mathrm{P}<0.05)$, but the GI value of type II glenoid cavities was larger than that of type III cavities $(\mathrm{P}<0.05)$. The $\mathrm{CD}$ value showed a difference between type I and type III glenoid cavities $(\mathrm{P}<0.05)$. For the EF parameter, the values of type III glenoid cavities were significantly larger than those of type I and II glenoid cavities $(\mathrm{P}<0.05)$.

Conclusions: Measuring and observing the variety of shapes and sizes of the glenoid cavity in Chinese people is conducive to for better understand its morphological features. This information can also guide surgeons in the design and selection of suitable prostheses for total shoulder arthroplasty in the Chinese population in order to reduce postoperative complications.

Key words: glenoid cavity, anatomic variation, classification, morphology, threedimensional reconstruction, glenoid notch 


\section{INTRODUCTION}

The shoulder joint, which consists of the eminence of the humerus and the glenoid cavity of the scapula, is one of the most flexible but unstable joints of the human body. Once the shoulder joint or scapula is damaged, the glenoid cavity might become injured as well. Hence, the diagnosis and treatment of many shoulder diseases are related to the glenoid cavity. Numerous studies have established that understanding the anatomical characteristics of the glenoid cavity is conducive to choosing the proper therapies for shoulder dislocation, scapular fractures and glenohumeral arthritis ${ }^{1-4}$ and especially for improving the surgical treatment for Bankart injury or anterior shoulder dislocation ${ }^{5}$. The morphology of the glenoid cavity should be considered when selecting the ideal anchor insertion angle for a Bankart repair ${ }^{6}$. The morphological structure of the glenoid cavity affects some common shoulder operative methods, such as total shoulder arthroplasty, in addition to associated diseases ${ }^{7-10}$. This structure can guide orthopedic surgeons to ensure the appropriate shape and size, as well as the individual placement of prosthetic components ${ }^{11}$. Therefore, it is important to investigate the anatomical morphology of the glenoid cavity in clinical practice.

To date, many papers have documented the anatomical characteristics and physiology of the glenoid cavity. Mamatha T et al. ${ }^{12}$ reported several anatomical parameters used to define the shape and dimensions of the glenoid cavity that were beneficial for designing glenoid prostheses in the South Indian population. Hina B Rajput et al. ${ }^{4}$ not only described the anatomy of the glenoid cavity but also created a classification based on its shape. In terms of the classification, most of the traditional anatomical standards have described the shape of the glenoid cavity as pear-shaped, round, or oval or as having an inverted comma shape. Then, some researchers incorporated the glenoid notch into one criterion for categorization ${ }^{13-14}$. Prescher A et al. ${ }^{13}$ explained how this notch would influence the shape of the glenoid cavity. They 
indicated that when the notch was present, the shape could be described as pear- or inverted-comma-shaped. When the notch was absent, the cavity was round or oval in shape. However, in general, most studies describing the morphological classification of the glenoid cavity have only focused on a single point, such as observing the glenoid notch, or have merely measured the anatomical parameters. Few studies have combined anatomical measurements and the glenoid notch to form the glenoid cavity, which can completely and quantitatively identify the certain morphology. Moreover, no studies have reported the morphological classification among Chinese people.

This study was performed to define a new morphological classification of the glenoid cavity in the Chinese population using three-dimensional (3D) reconstruction in order to better manage and promote relevant knowledge of shoulder diseases and surgical techniques.

\section{MATERIALS AND METHODS}

\section{Patients}

A total of 501 patients who underwent computed tomography (CT) scans of the unilateral shoulder in the period from 2017 to 2020 at the Department of Orthopedics, Chinese and Western Medicine Hospital of Yibing were identified (mean age 49.04 \pm 16.13 years). This study strictly adhered to the inclusion criteria and exclusion criteria. The study included 254 patients with scans of the right side (mean age 49.05 \pm 16.30 years, 114 females and 140 males), and the remaining patients had scans of the left side (mean age 49.02 \pm 15.99 years, 110 females and 137 males), with no significant difference in between the left and right side. Inclusion criteria: (1) The glenoid cavity was clear on each scan. Only normal computed tomography images were used. (2) The basic information and imaging data were complete. Exclusion criteria: (1) Any patient with a history of posterior or multidirectional instability, prior surgery, or glenohumeral arthritis. (2) Patients who had contraindications for CT. 


\section{D reconstruction of the scapula}

All measurements were carried out at the Radiology Department of the Department of Orthopedics, Chinese and Western Medicine Hospital of Yibing. A spiral CT scanner (Somatom Emotion; Siemens AG, Munich, Germany) was used, with a scanning voltage of $120 \mathrm{kV}$, tube current of $120 \mathrm{mAs}$, and pitch of 0.8 . Then, scanned images with 0.75 -mm thickness were reconstructed, with a reconstruction interval of $0.75 \mathrm{~mm}$. Next, 3D reconstructions were generated after CT scanning by the Glenosys software (Imascap; Brest; France) which is an automated segmentation process $^{15}$. All 3D images were stored in the Picture Archiving Communication System (PACS; DJ Health Union Systems Corporation, Shanghai, China).

\section{Measurement of the glenoid cavity}

After acquiring the 3D reconstruction models, measurements were made by 2 researchers, also referred to as observers, who had been engaged in the field of radiology for more than three years. These researchers independently obtained measurements, and each measurement was repeated three times; next, an average of the three values was obtained. In the case of any differences in the results, a 3rd medical practitioner was consulted.

Using the 3D reconstructed scapulae, three experienced researchers used the Glenosys software to manually extract the contour of the glenoid articular surface and points. This software could automatically recognize the morphological structure of the proximal humerus, scapula and glenoid. The superior point (highest point on the glenoid articular surface) and the inferior point (lowest point on the glenoid articular surface) were chosen by the Glenosys software program. Based on these extracted points, the glenoid articular surface was defined for each scapula. The first scapular coordinate system was defined by a center and three orthogonal vectors. The center 
(center of gravity of the glenoid articular surface) was automatically calculated. The three vectors were the vertical axis formed by the intersection of the scapula plane and the glenoid plane (oriented from lower to upper), the sagittal axis oriented back to front and perpendicular to the scapular plane, and the frontal axis oriental medial to lateral and perpendicular to the other two vectors. The second scapular coordinate system was similar to the first system. However, two vectors, the frontal axis oriented medial to lateral and perpendicular to the glenoid plane and the sagittal axis oriented back to front and perpendicular to the other two vectors were different. The scapular coordinate system was used to calculate height, width, height at max width and bestfit sphere by the Glenosys software program.

The following parameters were defined and measured (accurate to $0.1 \mathrm{~mm}$ ) in the $3 \mathrm{D}$ reconstruction models (Fig. 1).

(1) A: the most prominent point on the upper edge of the glenoid cavity.

(2) B: the lowest point of the glenoid cavity.

(3) C: in the upper part of the glenoid cavity, the posterior intersection of the longest line perpendicular to the line defining $\mathrm{AB}$ and the glenoid rim.

(4) D: in the upper part of the glenoid cavity, the anterior intersection of the longest line perpendicular to the line defining $\mathrm{AB}$ and the glenoid rim.

(5) E: in the lower part of the glenoid cavity, the posterior intersection of the longest line perpendicular to the line defining $\mathrm{AB}$ and the glenoid rim.

(6) F: in the lower part of the glenoid cavity, the anterior intersection of the longest line perpendicular to the line defining $\mathrm{AB}$ and the glenoid rim.

(7) AB: represents the superior-inferior glenoid diameter. (The line formed by A points connected to B points)

(8) CD: represents the upper anterior-posterior glenoid diameter. (The line formed by C points connected to D points) 
(9) EF: represents the lower anterior-posterior glenoid diameter. (The line formed by E points connected to F points)

(10) GI: represents the height-to-width ratio of the glenoid cavity (the ratio of $\mathrm{AB}$ to $\mathrm{EF})$.

\section{Statistical analysis}

All data were collected based on body side, sex and morphology. Morphological data are presented as the mean \pm standard deviation (SD). Statistical analysis was performed using SPSS 20.0 software (IBM Corp, Armonk, NY, USA). The significance level was set at $\mathrm{P}=0.05$.

Statistically significant differences in body side and sex were assessed using independent sample t-tests and Chi-square tests. Two-way ANOVA and GamesHowell tests were applied to analyse differences in the anatomic parameters of the glenoid cavity among sides, sexes and classifications.

\section{RESULTS}

\section{Morphological classification of the glenoid cavity}

The glenoid morphology was classified into five types based on the anatomical measurements and the shapes identified in previous literature; the shape was determined by the slightly raised rim of the glenoid cavity (Fig. 2).

According to a previous study, two criteria were used for this classification: one was the presence of the glenoid notch (located approximately somewhat above the middle of the anterior margin of the cavity) and the other was the comparison of the diameters of the upper and lower parts of the glenoid cavity (CD and EF). In addition, according to Prescher et al. [13], we recognized different types of notches were identified. The specific definitions of the five types are as follows. 
(1) type I: pear-shaped glenoid cavities (CD was less than EF, with a shallow notch at the anterior glenoid rim of the upper part of the glenoid cavity)

(2) type II: oval-shaped glenoid cavities (CD was approximately equal to EF, without a notch)

(3) type III: teardrop-shaped glenoid cavities (CD was less than EF, without a notch)

(4) type IV: calabash-shaped glenoid cavities (CD was less than EF, with two notches at the anterior and posterior glenoid rims of the upper part of the glenoid cavity)

(5) type V: inverted-comma-shaped glenoid cavities (CD was less than EF, with a prominent notch at the anterior glenoid rim of the upper part of the glenoid cavity)

Of the 501 patients, 53.29\% exhibited type I; 24.15\% exhibited type II and type III, type IV and type V were exhibited by $17.96 \%, 2.60 \%$ and $2.00 \%$, respectively. The incidence of type I (pear-shaped) cavities was the highest (53.29\%) among the total sample ( $n=501$ ), while the incidence of type IV (inverted-comma-shaped) was the lowest (2.00\%). There was no significant difference with regard to body side or sex ( $\mathrm{P}>0.05)$. The constituent ratios of the classifications by body side and sex are displayed in Table 1.

\section{Morphological parameters of the glenoid cavity}

The parameters obtained were not significantly different in terms of body side, except for the average CD value (the right side was $1.99 \pm 0.28 \mathrm{~cm}$, and the left side was $1.91 \pm 0.28 \mathrm{~cm}, \mathrm{P}=0.001)$. The significance of the mean values by sex was not surprising; all the average values, except for the GI value, were larger in males than in females $(\mathrm{P}=0.000)$.

Moreover, the parameters exhibited apparent differences among various glenoid 
cavity types. The AB and EF values of type II cavities $(3.40 \pm 0.45 \mathrm{~cm} ; 2.50 \pm 0.38 \mathrm{~cm})$ were the shortest when compared to the other four types, but the GI value ( $1.37 \pm 0.40$ $\mathrm{cm}$ ) was the longest. A statistically significant difference was found between type II and type III cavities ( $\mathrm{P}=0.005)$. The average $\mathrm{CD}$ value of type III cavities $(2.02 \pm 0.23$ $\mathrm{cm})$ was greater than that of type I cavities $(1.92 \pm 0.29 \mathrm{~cm})$, which was considered statistically significant $(\mathrm{P}=0.005)$ (Table 2$)$.

\section{DISCUSSION}

This study analysed the morphological features of the glenoid cavity using some defined anatomical parameters and five types of shapes to reveal the physiological characteristics of the glenoid cavity in the Chinese population. In particular, we found the calabash shape to be a new classification, which illustrates the differences between Chinese people and other populations. This is an improvement from previous studies in which similar measurements were made in other populations. On the one hand, when assessing a variety of shoulder diseases involving the glenoid cavity, our results can assist surgeons in formulating the appropriate treatment for native patients. The glenoid cavity has variations which means that some compensational mechanism must exist. However, the biomechanical mechanisms and anatomical parameters probably provide this compensation ${ }^{16}$. For total shoulder arthroplasty in the Chinese population, the calabash shape of the glenoid cavity may have to be taken into consideration when designing and fitting components.

Shoulder instability is a common problem in the general population and can be caused by multiple factors, including damage or dysfunction of the glenohumeral articulation, labrum, and glenohumeral ligaments ${ }^{17}$. The glenoid cavity is one of the most critical parts of the shoulder joint. Prescher A et al. ${ }^{13}$ proposed that the shape of the glenoid cavity impacts the attachment of the glenoid labrum. Furthermore, the design and placement of prostheses are related to the anatomic parameters of the glenoid cavity, such as its height, width, and shape ${ }^{18}$. Therefore, it is important to 
accurately understand the morphological classification and morphometrical data of the glenoid cavity.

Various studies have classified the glenoid cavity based on the presence of a notch in the anterior rim of the glenoid cavity. Anetzberger and Putz ${ }^{19}$ divided the glenoid cavity into teardrop-shaped (types Ia and Ib, with and without a notch) and elongated oval-shaped (type II) cavities, but descriptions of these shapes are rare. Prescher A et al. ${ }^{13}$ classified the glenoid cavity as pear-shaped (55\%) (type I with a distinct glenoid notch and type 2 with a slight notch) and oval-shaped (45\%) cavities. Subsequently, a glenoid cavity with a distinct notch and an inverted-comma shaped was proposed separately. A majority of studies classified the shape of the glenoid cavity as pear-shaped, inverted-comma-shaped or oval-shaped cavity ${ }^{4,12,19}$. However, we found that there still existed another shape existed, a calabash-shaped cavity, in addition to the four shapes mentioned above. The key point for distinguishing this type of shape is the difference between it and common shapes. With common shape, a notch is either present or absent in the anterior rim of the glenoid cavity, while calabash-shaped cavities have two notches in the anterior and posterior glenoid rims.

The five types of glenoid cavities that our study described are based on the Chinese population. Type I (pear-shaped) cavities exhibited the the maximum frequency of 53.29\% in all cases, but type IV (inverted comma-shaped) cavities exhibited the minimum frequency of $2.00 \%$. In terms of other populations, many studies have also shown that pear-shaped cavities are the most common ${ }^{13,20-21}$. However, Mamatha T et al. ${ }^{12}$ and Rajput HB et al. ${ }^{4}$ who studied the Indian population, proposed that oval-shaped glenoid cavities oval in shape occurred less frequently than those with other shapes, which was different from the results of our study. Nevertheless, the types of glenoid cavities in the Egyptian population according to ElDin WA and Ali $\mathrm{MH}^{20}$ were consistent with the types found in our study. Additionally, we analysed the average values of the glenoid cavity in terms of body side and sex. According to our results, the values of the right glenoid cavity, except for the CD value were higher than those of the left glenoid cavity, but the difference was not 
statistically significant ( $\mathrm{p}=0.250$ ); this finding shows that the size of the right glenoid cavity is likely similar to that of the left glenoid cavity. The average values of both the right and left glenoid cavities were larger in our study than in the studies by Mamatha T et al. ${ }^{12}$ and Rajput HB et al. ${ }^{4}$ but were smaller than the results of El-Din WA and Ali $\mathrm{MH}^{20}$. In addition, the significance of the mean values in terms of sex was similar to that of other studies; all measurements except the GI value were larger in males than in females. We also considered the mean values of the glenoid cavity among the different classifications and found that the $\mathrm{AB}$ value was the shortest in type II but the GI value was the largest among the other four types. A statistically significant difference was only found between type II and type III glenoid cavities. Brett-D Owens et $\mathrm{al}^{22}$ found that tall and thin glenoid cavities were associated with a higher risk of anterior instability than short and wide glenoid cavities, indicating that type II glenoid cavities might more frequently lead to anterior instability more frequently than type III glenoid cavities. For type III glenoid cavities, the CD value was the largest and the EF value was the widest among the five types. These differences indicate that the shape and size of the glenoid cavity are associated with geographic region and race, and that understanding these features is conducive to the individualization of surgical treatment.

The present study provides a comprehensive classification of the glenoid cavity in the Chinese population. One of the main contributions of the study is the proposal of a new classification in terms of the calabash shape. To our knowledge, many studies have focused on glenoid classifications in different populations, but few have focused on glenoid classifications in the Chinese population. Furthermore, previous studies have mostly used dry specimens, which are smaller in size, have an unknown sex and have a higher number of defects ${ }^{12,19}$, to investigate the glenoid classification of the glenoid cavity, and these factors may influence the conclusion. Thus, to solve these problems, our study included a large samples size $(n=501)$ and used CT scans in order to reduce the experimental error caused by an insufficient sample size. In addition, knowledge of the sex of the included patients was beneficial for analyzing 
sex differences. Moreover, use of 3D reconstruction in research can accurately reflect the actual anatomy of the glenoid cavity ${ }^{23}$.

This study has some limitations. Osteoarthritic glenoid cavities were not examined, and thus, the ideal prosthesis size was not determined. In addition, the height of the patients was not recorded, which is associated to the dimensions of the scapula. Although 3D models were reconstructed, these parameters were only measured on 2D images. Descriptive parameters were researched but the variability and seek for volumes or forms were not shown.

\section{CONCLUSIONS}

By measuring and observing the shape of the glenoid cavity in Chinese people, the present study divided the shape into five types, including a new classification of calabash-shaped cavity. These anatomical parameters and classifications will help to better understand the morphological features of the glenoid, design and select suitable prostheses for total shoulder arthroplasty in the Chinese population.

\section{Compliance with ethical standards}

All procedures were allowed by Department of Orthopedics, Chinese and Western Medicine Hospital of Yibing with the following reference number: KY2020056.

\section{Acknowledgments}

The authors would like to thank all patients who agreed to participate in this study and the Department of Orthopedics, Chinese and Western Medicine Hospital of Yibing for providing the PACS CT system. 


\section{Funding}

This study was supported by Yibin Scientific Research and Technology Bureau Project, grant number 2020SF005; Yibin Municipal Health Commission Research Project, grant number 2020YW007.

Conflict of interest: None declared

\section{REFERENCES}

1. Friedman RJ, Hawthorne KB, Genez BM. The use of computerized tomography in the measurement of glenoid version. J Bone Joint Surg Am. 1992, 74(7):10327.

2. F. Gilbert, L. Eden, R. Meffert, et al. Intra- and interobserver reliability of glenoid fracture classifications by Ideberg, Euler and AO. BMC Musculoskeletal Disorders. 2018, 19(1):89.

3. Petr Fulin, Martin Kysilko, David Pokorny, et al. Study of the variability of scapular inclination and the glenoid version-considerations for preoperative planning: clinical-radiological study. BMC Musculoskeletal Disorders.2017, 18(1):16.

4. Rajput HB, Vyas KK, Shroff BD. A study of morphological patterns of glenoid cavity of scapula. National J Med Rese. 2012, 2:504-507.

5. Klemt C, Toderita D, Nolte D, et al. The critical size of a defect in the glenoid causing anterior instability of the shoulder after a Bankart repair, under physiological joint loading. Bone Joint J. 2019, 101-B (1):68-74.

6. Grieshober JA, Palmer JE, Kim H, et al. Comparison of Curved and Straight Anchor Insertion for Bankart Repair. Orthopedics. 2019, 42(2): e242-e246.

7. Alentorn-Geli E, Samitier G, Torrens C, Wright TW. Reverse shoulder 
arthroplasty. Part 2: Systematic review of reoperations, revisions, problems, and complications. Int J Shoulder Surg. 2015, 9(2):60-7.

8. Hawi N, Reinhold A, Suero EM, et al. The anatomic basis for the arthroscopic Latarjet Procedure: A cadaveric study. Am J Sport Med. 2016, 44(2):497-503.

9. Longo UG, Petrillo S, Berton A, Denaro V. Reverse total shoulder arthroplasty for the management of fractures of the proximal humerus: a systematic review. Musculoskelet Surg. 2016, 100(2):83-91.

10. Merkle TP, Beckmann N, Bruckner T, Zeifang F. Shoulder joint replacement can improve quality of life and outcome in patients with dysmelia: a case series. BMC Musculoskelet Disord. 2016, 17:185-193.

11. Zumstein V, Kraljevic M, Hoechel S, Conzen A, Nowakowski AM, Muller-Gerbl M. The glenohumeral joint-a mismatching system? A morphological analysis of the cartilaginous and osseous curvature of the humeral head and the glenoid cavity. J Orthop Surg Res. 2014, 9:34-39.

12. Mamatha T, Pai SR, Murlimanju BV, Kalthur SG, Pai MM, Kumar B. Morphometry of Glenoid Cavity. Online J Health Allied Scs. 2011, 10.

13. Prescher A, Klumpen T. The glenoid notch and its relation to the shape of the glenoid cavity of the scapula. J Anat. 1997, 190 (Pt 3):457-60.

14. Huber CE. Zur Form und GrÖße der Cavitas glenoidalis. Anatomischer Anzeiger. 1991, 172:137-142.

15. Boileau P, Gauci MO, Wagner ER, Clowez G, Chaoui J, Chelli M, Walch G. The reverse shoulder arthroplasty angle: a new measurement of glenoid inclination for reverse shoulder arthroplasty. J Shoulder Elbow Surg. 2019, 28(7): 1281-1290.

16. De Wilde LF. CORR Insights ${ }^{\circledR}$ : The Muscle Cross-sectional Area on MRI of the Shoulder Can Predict Muscle Volume: An MRI Study in Cadavers. Clin Orthop Relat Res. 2020, 478(4): 884-885. 
17. Peter S, MD Chad R, MD Kristofer J, MD Frank A. Glenohumeral Dislocation Arthropathy: Etiology, Diagnosis, and Management. J Am Acad Orthop Surg. 2018, 00:1-9.

18. Schrumpf M, Maak T, Hammoud S, Craig EV. The glenoid in total shoulder arthroplasty. Curr Rev Musculoskelet Med. 2011, 4:191-199.

19. Anetzberger H, Putz R. The scapular: principle of construction and stress. Acta anatomica. Acta Anat (Basel). 1997, 156(1):70-80.

20. El-Din WA, Ali MH. A Morphometric Study of the Patterns and Variations of the Acromion and Glenoid Cavity of the Scapulae in Egyptian Population. J Clin Diagn Res. 2015, 9(8):AC08-11.

21. Checroun AJ, Hawkins C, Kummer FJ, Zuckerman JD. Fit of current glenoid component designs: An anatomic cadaver study. J Shoulder Elbow Surg. 2002, 11(6):614-7.

22. Brett-D Owens, Scot-E Campbell, Kenneth-L Cameron. Risk factors for anterior glenohumeral instability. The American journal of sports medicine. 2014, 42:2591-2596.

23. Moineau G, Levigne C, Boileau P, Young A, Walch G. Three-dimensional measurement method of arthritic glenoid cavity morphology: feasibility and reproducibility. Orthop Traumatol Surg Res. 2012, 98(6 Suppl): S139-45.

Table 1. Sample size and constituent ratio of the glenoid cavity based on classification 


\begin{tabular}{llllll}
\hline Classification & \multicolumn{2}{c}{ Side } & \multicolumn{2}{c}{ Gender } & Total \\
\cline { 2 - 6 } & Right $^{\mathrm{a}}$ & Left & Male $^{\mathrm{b}}$ & Female & \\
& $\mathrm{N}(\%)$ & $\mathrm{N}(\%)$ & $\mathrm{N}(\%)$ & $\mathrm{N}(\%)$ & $\mathrm{N}(\%)$ \\
\hline Type I & $133(52.36)$ & $134(54.25)$ & $144(51.99)$ & $123(54.91)$ & $267(53.29)$ \\
Type II & $69(27.17)$ & $52(21.05)$ & $65(23.47)$ & $56(25.00)$ & $121(24.15)$ \\
Type III & $43(16.93)$ & $47(19.03)$ & $55(19.85)$ & $35(15.63)$ & $90(17.96)$ \\
Type IV & $4(1.57)$ & $9(3.64)$ & $7(2.52)$ & $6(2.68)$ & $13(2.60)$ \\
Type V & $5(1.97)$ & $5(2.03)$ & $6(2.17)$ & $4(1.78)$ & $10(2.00)$ \\
\hline
\end{tabular}

Note: a: P > 0.05 Vs Left, b: P > 0.05 Vs Female

Table 2. Mean values of the glenoid diameters (mean \pm SD)

\begin{tabular}{|c|c|c|c|c|c|}
\hline \multicolumn{2}{|c|}{ Parameters } & \multirow{2}{*}{$\begin{array}{l}\mathrm{AB}(\mathrm{cm}) \\
3.53 \pm 0.39\end{array}$} & \multirow{2}{*}{$\begin{array}{c}\mathrm{CD}(\mathrm{cm}) \\
1.99 \pm 0.28^{\mathrm{a}}\end{array}$} & \multirow{2}{*}{$\begin{array}{l}\mathrm{EF}(\mathrm{cm}) \\
2.62 \pm 0.33\end{array}$} & \multirow{2}{*}{$\begin{array}{l}\mathrm{GI}(\mathrm{cm}) \\
1.35 \pm 0.11\end{array}$} \\
\hline Side & Right & & & & \\
\hline & Left & $3.49 \pm 0.43$ & $1.91 \pm 0.28$ & $2.58 \pm 0.34$ & $1.36 \pm 0.12$ \\
\hline & $P$ & 0.298 & 0.001 & 0.25 & 0.772 \\
\hline \multirow[t]{3}{*}{ Gender } & Male & $3.71 \pm 0.38^{\mathrm{b}}$ & $2.06 \pm 0.26^{\mathrm{b}}$ & $2.76 \pm 0.31^{b}$ & $1.35 \pm 0.12$ \\
\hline & Female & $3.25 \pm 0.28$ & $1.81 \pm 0.23$ & $2.40 \pm 0.25$ & $1.36 \pm 0.11$ \\
\hline & $P$ & 0.000 & 0.000 & 0.000 & 0.270 \\
\hline \multicolumn{2}{|c|}{ Classification Type I } & $3.52 \pm 0.40^{c}$ & $1.92 \pm 0.29^{\mathrm{d}}$ & $2.61 \pm 0.33^{\mathrm{cd}}$ & $1.36 \pm 0.40$ \\
\hline & Type II & $3.40 \pm 0.45$ & $1.96 \pm 0.30$ & $2.50 \pm 0.38$ & $1.37 \pm 0.12$ \\
\hline
\end{tabular}




\begin{tabular}{|c|c|c|c|c|c|}
\hline & Type III & $3.60 \pm 0.36^{c}$ & $2.02 \pm 0.23$ & $2.72 \pm 0.26^{c}$ & $1.33 \pm 0.10^{c}$ \\
\hline & Type IV & $3.46 \pm 0.29$ & $1.89 \pm 0.23$ & $2.60 \pm 0.37$ & $1.34 \pm 0.13$ \\
\hline & Type V & $3.68 \pm 0.37^{c}$ & $1.87 \pm 0.23$ & $2.71 \pm 0.34$ & $1.36 \pm 0.09$ \\
\hline Total & & $3.51 \pm 0.41$ & $1.95 \pm 0.28$ & $2.60 \pm 0.34$ & $1.35 \pm 0.12$ \\
\hline
\end{tabular}

Note: a: $\mathrm{P}<0.05$ vs. Right; b: $\mathrm{P}<0.001$ vs. Female; c: $\mathrm{P}<0.05$ vs. Type $\mathbb{I}$; d: $\mathrm{P}<$ 0.05 vs. Type III

Figure 1. Schematic diagram showing diameters of the glenoid cavity. AB: represents the superior-inferior glenoid diameter. CD: represents the upper anterior-posterior glenoid diameter. EF: represents the lower anterior-posterior glenoid diameter.

Figure 2. Classification of glenoid cavity. (a) Type I is pear shaped. (b) Type II means is oval shaped. (c) Type III is teardrop shaped. (d) Type IV is calabash shaped. (e) Type $\mathrm{V}$ is inverted comma shaped. 


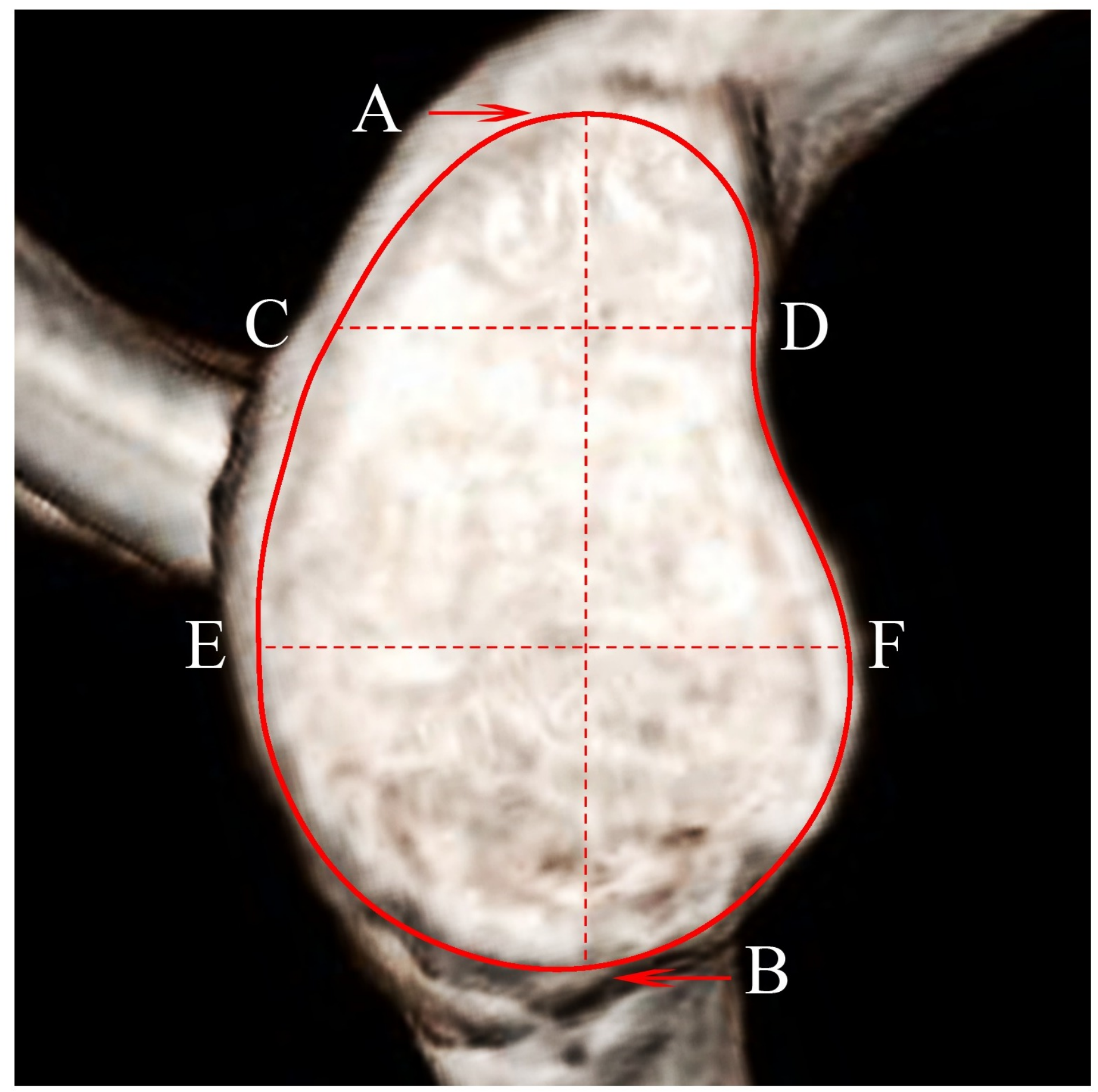




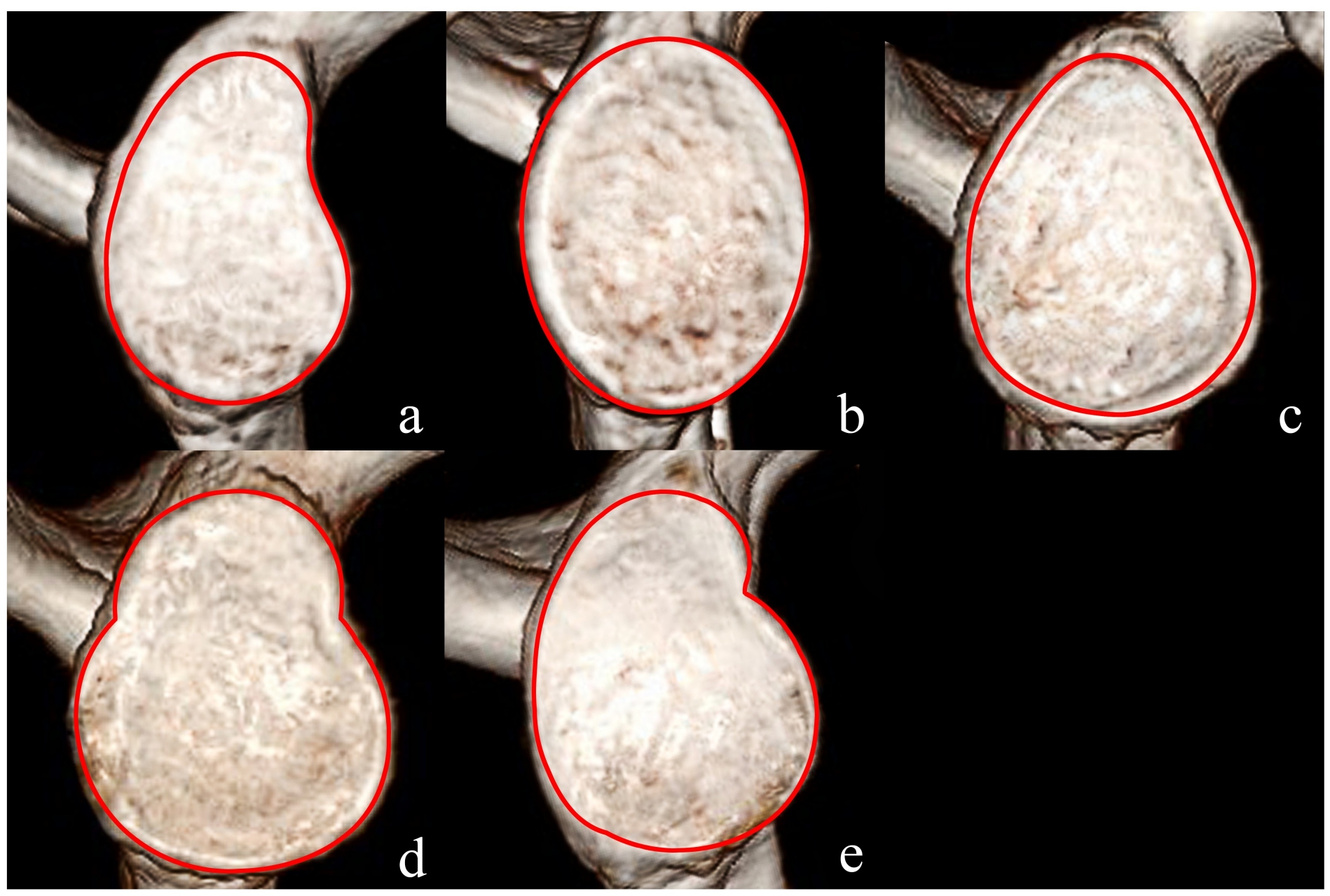

